\title{
Development and validation of a prognostic nomogram for early HER2-positive and lymph node-negative breast cancer
}

\author{
Qiyun Shi ${ }^{1}$, Ju Wang ${ }^{2}$, Xiang Ai ${ }^{1}$, Juncheng Xuhong ${ }^{1}$, Dandan Ma ${ }^{1}$, Yi Zhang ${ }^{1}$, Xiaowei Qi ${ }^{1}$ Jun Jiang ${ }^{1}$ \\ ${ }^{1}$ Department of Breast and Thyroid Surgery, Southwest Hospital, Army Medical University, Chongqing, China; ${ }^{2}$ Chongqing Municipal Center for \\ Disease Control and Prevention, Chongqing, China \\ Contributions: (I) Conception and design: X Qi, J Jiang, Q Shi; (II) Administrative support: J Xuhong, D Ma, Y Zhang; (III) Provision of study \\ materials or patients: None; (IV) Collection and assembly of data: Q Shi, J Wang; (V) Data analysis and interpretation: Q Shi, J Wang, X Ai; (VI) \\ Manuscript writing: All authors; (VII) Final approval of manuscript: All authors. \\ Correspondence to: Jun Jiang, MD, PhD; Xiaowei Qi, MD, PhD. Department of Breast and Thyroid Surgery, Southwest Hospital, Army Medical \\ University, Chongqing 400038, China. Email: jcbd@medmail.com.cn; qxw9908@foxmail.com.
}

Background Dual-targeted therapy is currently the standard adjuvant treatment for human epidermal
growth factor receptor 2-positive (HER2+) and lymph node-positive (LN+) breast cancer. However, the
optimal therapeutic strategy for patients with HER2+ and lymph node-negative (LN-) breast cancer remains
unclear. This population-based study aimed to explore the factors associated with survival in patients with
HER2+ and LN- breast cancer, and develop a survival-predicting nomogram in the era of trastuzumab-
based single-targeted therapy.
Methods: We collected the clinicopathological information of HER2+ and LN- breast cancer patients
who underwent chemotherapy and surgery from The Surveillance, Epidemiology, and End Results (SEER)
database (2010-2016, the Trastuzumab-based single-targeted therapy era). We subsequently explored the
risk factors for breast cancer-specific survival (BCSS) and overall survival (OS) using a Cox proportional
hazards regression model, aiming to identify subgroups with worse prognosis, which would indicate potential
demand for dual-targeted therapy. Three- and 5-year survival probability-predictive nomograms were
established and subjected to bootstrap internal validation. The concordance index (C-index) and calibration
curve were applied to evaluate the performance of the model. Results: After data cleansing, a total of 13,755 patients were included in the current analysis. Using univariate and multivariate Cox proportional hazards regression, higher clinical $\mathrm{T}$ stage, hormone receptorsnegative (HR-), and partial mastectomy without radiotherapy were identified as independent risk factors for BCSS and OS in patients with HER2 + and LN- breast cancer. Nomograms for 3- and 5-year BCSS and OS incorporating the selected prognostic factors were established. Calibration curves verified good consistency between the actual and nomogram-predicted survival probability. The C-index values of the BCSS and OS predictions and 95\% confidence interval (CI) were $0.773(0.740-0.806)$ and $0.764(0.737-0.791)$, respectively. Conclusions: Higher clinical T stage, HR-, and partial mastectomy without radiotherapy predicted worse prognosis in patients with HER2 + and LN- breast cancer. In clinical practice, patients can be recommended for single-targeted or dual-targeted therapy according to the individualized factors.

Keywordsa Breast cancer; HER2-positive; lymph node-negative; nomogram; survival analysis

Submitted May 25, 2021. Accepted for publication Jul 14, 2021.

doi: $10.21037 /$ gs-21-392

View this article at: https://dx.doi.org/10.21037/gs-21-392

^ ORCID: 0000-0003-3062-8846. 


\section{Introduction}

Approximately $15-20 \%$ of all patients with breast cancer overexpress human epidermal growth factor receptor 2 (HER2), which was considered a risk factor associated with disease aggression and reduced response to traditional chemotherapy before the advent of HER2-directed therapies (1). In the era of anti-HER2 targeted therapy, the prognosis of HER $2+$ breast cancers has markedly improved (2-8). Trastuzumab, an anti-HER2 humanized monoclonal antibody, was approved by the U.S. Food and Drug Administration (FDA) in 1998. Ever since, it has become an anti-HER2 treatment choice, regardless of early-stage or metastatic breast cancer (4,9-12). However, despite the significant improvements realized by singletargeted therapy, a considerable number of HER2+ patients with inherent and acquired resistance to trastuzumab will still suffer relapse and disease progression (3,5,11,13-15). Numerous attempts with novel approaches to target therapy have been performed to improve outcomes for patients with early HER2 + breast cancer (16).

The phase III APHINITY trial (NCT01358877) proved that pertuzumab significantly improved the rates of invasive-disease-free survival among patients with HER2+ operable breast cancer when added to trastuzumab and chemotherapy. Hence, the combination of pertuzumab plus trastuzumab and chemotherapy was approved in 2017 by the U.S. FDA for the adjuvant treatment of patients with early HER2+ breast cancer with high risk of recurrence $(17,18)$. However, in the APHINITY trial, in the cohort of patients with node-negative breast cancer, the 3-year rate of invasive-disease-free survival (iDFS) of patients in the pertuzumab group did not show significant improvement compared to those in the placebo group (hazard ratio, 1.13; 95\% CI, 0.68-1.86; $\mathrm{P}=0.64$ ) (17), which was confirmed by a long-term follow-up in 2019 (19).

Also, the phase III ExteNET trial (NCT00878709) confirmed the efficacy of neratinib, a tyrosine kinase inhibitor (TKI) of HER1, HER2, and HER4, after trastuzumab-based adjuvant therapy in patients with earlystage HER2 + breast cancer (20-22). The concurrent or sequential treatment of neratinib within 1 year after trastuzumab adjuvant therapy significantly improved the 5-year iDFS and 8-year overall survival (OS) of HER2+ breast cancer patients. However, in the subgroup analysis of the ExteNET trial, the LN- cohort $(n=255)$ did not show significant improvement in the 5-year iDFS (hazard ratio,
0.37; 95\% CI, 0.08-1.24) (22), which was similar to the APHINITY trial. Neither APHINITY trial and ExteNET trial support of the routine use of dual-targeted treatment over HER2 + and LN- breast cancer, hence it remains controversial whether dual-targeted therapy is suitable for HER2+ and LN- breast cancer patients.

Therefore, we used the data of HER2+ and LN- breast cancer patients who were diagnosed between 2010 and 2016 (before dual-targeted therapy was approved), and designed a protocol to screen out risk factors associated with survival outcomes in this population. Furthermore, we established and validated prognostic nomograms, in an attempt to identify subgroups with poorer survival outcomes, indicating the potential demand of dual-targeted therapy (trastuzumab plus pertuzumab or TKIs, or sequential TKIs after trastuzumab therapy). We present the following article in accordance with the TRIPOD reporting checklist (available at https://dx.doi.org/10.21037/gs-21-392).

\section{Methods}

\section{Data source and patient selection}

All procedures performed in the study involving human participants were in accordance with Helsinki declaration (as revised in 2013). Data for this study were selected from 18 registries of the Surveillance, Epidemiology, and End Results (SEER) program, and obtained using SEER*Stat software version 8.3.6 (23). The target population was extracted based on the following criteria in the SEER database: (I) patients diagnosed between January 1, 2010 and December 31, 2016; (II) female patients; (III) histologically confirmed malignant breast cancer; (IV) known primary tumor status and not carcinoma in situ; (V) first and primary tumor without distant metastasis; (VI) HER2 + and LN- subtype; (VII) patients who underwent chemotherapy and cancer-directed surgery; (VIII) survival data with complete and available dates, and more than 0 days of survival; (IX) patients with medical insurance; (X) clear demographics and clinicopathological information available for all variables of interest including age at diagnosis, race, laterality, grade, clinical $\mathrm{T}$ stage, hormone receptor (HR) status, radiotherapy status, and surgery type. Since chemotherapy plus surgery is the standard treatment for patients with non-metastatic operable breast cancer $(24,25)$, only those that underwent both chemotherapy and surgery were included. 


\section{Variables}

The primary endpoints were breast cancer-specific survival (BCSS) and OS. BCSS was defined as the time from the date of diagnosis until death due to breast cancer. OS was defined as the time from the date of diagnosis until death due to any cause. The cut-off date was December 31, 2016, which was the last update on follow-up time.

The following variables were selected as potential prognostic factors: age at diagnosis; ethnicity [White, Black, and other (American Indian/Alaska Native, Asian/ Pacific Islander)]; laterality (left or right); pathological grade (well-differentiated, moderately differentiated, poorly differentiated); clinical $\mathrm{T}$ stage (T1, T2, T3, and T4); HR status (ER+/PR+, ER+/PR-, ER-/PR+, ER-/PR-); radiotherapy status (yes or none/unknown); and surgery type (mastectomy or partial mastectomy). Clinical Tumor, lymph node and metastasis (TNM) stage was not included because of its collinearity with $\mathrm{T}$ stage under circumstances of $\mathrm{N} 0$ and $\mathrm{M} 0$.

\section{Statistical analysis}

Descriptive results were reported as counts and percentages for categorical variables, and as means \pm standard deviations for continuous variables. Kruskal-Wallis and Chi-squared tests were used to examine differences between the patient groups. A univariate Cox proportional hazards regression model was employed to analyze the independent variables associated with BCSS and OS. Variables with statistical significance $(\mathrm{P}<0.05)$ in the univariate analysis were included in the multivariate Cox proportional hazards regression model to estimate the adjusted hazard ratios (HRs) with $95 \%$ confidence interval (CI) of risk factors and to construct the nomograms. Survival results were generated using the Kaplan-Meier method and were compared using log-rank tests. A nomogram was built to predict the 3- and 5-year prognoses of BCSS and OS (26).

To validate the model, Harrell's concordance index (C-index) was introduced to calculate the discrimination between the actual result and the predicted result of the Cox survival analysis (27). The nomogram was validated internally by 1000 bootstrap resamples and by plotting calibration curves that compared the actual survival probability with predicted survival probability generated by the bootstrapping. 1000 bootstrap repetitions were chosen since higher repetition times can hardly improve the estimates.
$\mathrm{P}<0.05$ was considered to indicate statistical significance, and all $\mathrm{P}$ values were two-sided. All statistical analyses were conducted using SAS version 9.4 (SAS Institute Inc., Cary, NC, United States) and R 4.0.2 (R Foundation, https:// www.r-project.org/).

\section{Results}

\section{Patients' characteristics}

The patients' demographics and clinicopathologic features are summarized in Table 1. In total, 13,755 patients with early HER2+ and LN- breast cancer were enrolled in this study. Among these, 209 patients had breast cancer-specific deaths, and 325 patients died due to all causes. The average age of patients was 55.82 years [interquartile range (IQR), 48-64 years].

Only a minority of patients had pathological grade I tumors $(4.6 \%)$, while grade II $(37.0 \%)$ and grade III (58.4\%) tumors accounted for the majority of cases. Most patients had a stage T1 primary tumor $(60.2 \%)$, and the proportion decreased progressively with higher $\mathrm{T}$ stages. In terms of hormone receptors, estrogen receptor-positive $(\mathrm{ER}+) /$ progesterone receptor-positive $(\mathrm{PR}+)$ accounted for approximately half of the total $(51.7 \%)$, whereas the proportions were $20.2 \%$ for ER+/PR- or ER-/PR+ and $28.1 \%$ for ER-/PR-. With regards to the choice of surgery type and radiotherapy, the majority of patients received mastectomy without radiotherapy $(36.7 \%)$ or partial mastectomy with radiotherapy $(45.2 \%)$, which was consistent with the clinical consensus (28).

\section{Survival outcomes}

The 3-year survival rate of all patients was $97.9 \%$ (95\% CI, 97.6-98.2\%), and the 5-year survival rate was $95.7 \%$ (95\% CI, 95.2-96.3\%). After an unadjusted survival analysis, age group, ethnicity, grade, T stage, HR, and therapy exhibited a significant association with either BCSS or OS (Table 2), and were included in the multivariate Cox proportional hazards regression model. Forest plots of HR in the multivariate Cox analysis are shown in Figure 1A,B.

Among the different age groups, the $>80$ years old group had a significantly higher risk than the younger reference group both in terms of BCSS (HR $=5.22,95 \%$ CI: 1.19 22.83, $\mathrm{P}=0.028$ ) and OS (HR $=6.20,95 \% \mathrm{CI}: 1.87-20.58$, $\mathrm{P}=0.003)$. As for ethnicity, American Indian/Alaska Native/ Asian/Pacific Islander patients had a significantly better 
Table 1 Demographics and clinicopathologic characteristics of the entire cohort

\begin{tabular}{lc}
\hline Variable & $\mathrm{N}=13,755$ \\
\hline Age, mean (SD), years & $55.82(11.65)$ \\
Ethnicity, n (\%) & \\
White & $10,576(76.9)$ \\
Black & $1,506(10.9)$ \\
Other* & $1,673(12.2)$ \\
Laterality, n (\%) & \\
Left & $6,975(50.7)$ \\
Right & $6,780(49.3)$ \\
Grade, $\mathrm{n}(\%)$ & \\
I & $639(4.6)$ \\
II & $5,087(37.0)$ \\
III & $8,029(58.4)$ \\
T stage, $\mathrm{n}(\%)$ & \\
1 & $8,274(60.2)$ \\
2 & $4,819(35.0)$ \\
3 or 4 & $662(4.8)$
\end{tabular}

Hormone receptors, n (\%)

$\mathrm{ER}+/ \mathrm{PR}+$

7,105 (51.7)

$\mathrm{ER}+/ \mathrm{PR}-$ or ER-/PR+

$2,779(20.2)$

ER-/PR-

$3,871(28.1)$

Therapy, n (\%)

Only mastectomy

$5,054(36.7)$

Only partial mastectomy

$1,808(13.1)$

Mastectomy + radiotherapy

$678(4.9)$

Partial mastectomy + radiotherapy

$6,215(45.2)$

*, American Indian/Alaska Native, Asian/Pacific Islander. SD, standard deviation; ER, estrogen receptor; PR, progesterone receptor.

survival in terms of BCSS (HR $=0.48,95 \%$ CI: $0.26-0.90$, $\mathrm{P}=0.021)$ and $\mathrm{OS}$ (HR $=0.60,95 \%$ CI: $0.39-0.93, \mathrm{P}=0.022$ ) compared to White patients. T2 patients ( $\mathrm{HR}=1.85,95 \%$ CI: $1.46-2.34, \mathrm{P}<0.001)$ and $\mathrm{T} 3$ or $\mathrm{T} 4$ patients $(\mathrm{HR}=2.38$, $95 \%$ CI: $1.55-3.66, \mathrm{P}<0.001)$ had a significantly higher risk of total cause of death than T1 patients. The ER+/PR+ HR status exhibited the lowest risk among the four types, while the ER-/PR- patients had a significantly poorer BCSS (HR
$=2.23,95 \%$ CI: $1.58-3.15, \mathrm{P}<0.001)$ and $\mathrm{OS}(\mathrm{HR}=1.46$, 95\% CI: $1.13-1.90, \mathrm{P}=0.004)$. Patients that underwent partial mastectomy without radiotherapy ( $\mathrm{HR}=1.95,95 \%$ CI: $1.45-2.61, \mathrm{P}<0.001)$ and mastectomy with radiotherapy $(\mathrm{HR}=1.70,95 \% \mathrm{CI}: 1.12-2.59, \mathrm{P}=0.013)$ showed significantly higher risk, while patients that underwent partial mastectomy with radiotherapy had a lower risk (HR $=0.69,95 \%$ CI: $0.52-0.91, \mathrm{P}=0.008)$ of all cause death than those that underwent mastectomy without radiotherapy. By comparing the clinicopathological parameters of patients that underwent different therapies, we found that those who underwent mastectomy with radiotherapy had a significantly higher proportion of clinical T3 or T4 stages (Table S1), which may explain their higher risk of death. Significance associations were not observed in the other variables.

Figure 2 displays the OS curves of the total population and subgroups divided by clinicopathological parameters.

\section{Construction and validation of prognostic nomograms}

The nomograms of 3- and 5-year BCSS and OS incorporating the selected prognostic factors were established (Figure $3 A, B$ ). The calibration plots verified good consistency between the actual and nomogrampredicted survival probability in terms of both BCSS and OS (Figure 4). The C-index of BCSS and OS predictions were 0.773 (95\% CI, $0.740-0.806)$ and 0.764 (95\% CI, $0.737-0.791)$, respectively.

\section{Discussion}

In this population-based study, we identified several independent risk factors associated with the survival of HER2 + and LN- breast cancer patients, and developed and validated prognostic nomograms for predicting survival. To the best of our knowledge, this is first observational research exploring the prognostic factors of HER2+ and $\mathrm{LN}$ - populations in the H-based single-targeted therapy era, which can, to some extent, provide a reference for precision treatment in the era of dual-targeted therapy.

Although H-based, single-targeted therapy has considerably improved the prognosis of HER $2+$ breast cancer patients, inevitable recurrence and metastasis in some populations several years after treatment of earlystage disease necessitates intensive treatment $(3,5,11,13-15)$. The APHINITY and KAITLIN trials established dualtargeted therapy as adjuvant treatment in HER2+ and LN+ breast cancer; however, the optimal choice for patients with 
Table 2 Univariate Cox analysis of BCSS and OS

\begin{tabular}{|c|c|c|c|c|c|c|}
\hline \multirow{3}{*}{ Variables } & \multicolumn{3}{|c|}{ BCSS } & \multicolumn{3}{|c|}{ OS } \\
\hline & \multirow{2}{*}{$\frac{\text { Log-rank test }}{\mathrm{P} \text { value }}$} & \multirow{2}{*}{$\begin{array}{c}\text { Univariate analysis } \\
\text { HR }(95 \% \mathrm{Cl})\end{array}$} & \multirow{2}{*}{$P$ value } & \multirow{2}{*}{$\frac{\text { Log-rank test }}{\mathrm{P} \text { value }}$} & \multirow{2}{*}{$\begin{array}{c}\text { Univariate analysis } \\
\text { HR }(95 \% \mathrm{Cl})\end{array}$} & \multirow{2}{*}{$P$ value } \\
\hline & & & & & & \\
\hline Age group (years) & $<0.001$ & & & $<0.001$ & & \\
\hline $18-30$ & & Reference & & & Reference & \\
\hline $40-50$ & & $0.54(0.13,2.27)$ & 0.397 & & $0.48(0.15,1.56)$ & 0.221 \\
\hline $50-60$ & & $0.81(0.20,3.34)$ & 0.775 & & $0.76(0.24,2.41)$ & 0.642 \\
\hline $60-70$ & & $0.92(0.22,3.80)$ & 0.912 & & $1.26(0.40,3.98)$ & 0.69 \\
\hline $70-80$ & & $1.62(0.39,6.77)$ & 0.508 & & $2.52(0.79,7.99)$ & 0.117 \\
\hline White & & Reference & & & Reference & \\
\hline Black & & $1.22(0.81,1.86)$ & 0.342 & & $1.18(0.85,1.63)$ & 0.319 \\
\hline Other ${ }^{*}$ & & $0.48(0.26,0.88)$ & 0.018 & & $0.56(0.36,0.86)$ & 0.009 \\
\hline Laterality & 0.970 & & & 0.349 & & \\
\hline Left & & Reference & & & Reference & \\
\hline Right & & $0.99(0.75,1.33)$ & 0.97 & & $0.90(0.72,1.12)$ & 0.349 \\
\hline Grade & 0.013 & & & 0.024 & & \\
\hline I & & Reference & & & Reference & \\
\hline 3 or 4 & & $4.69(2.96,7.44)$ & $<0.001$ & & $3.50(2.40,5.09)$ & $<0.001$ \\
\hline Hormone receptors & $<0.001$ & & & $<0.001$ & & \\
\hline $\mathrm{ER}+/ \mathrm{PR}+$ & & Reference & & & Reference & \\
\hline $\mathrm{ER}+/ \mathrm{PR}-$ or ER-/PR+ & & $1.68(1.12,2.51)$ & 0.012 & & $1.52(1.14,2.02)$ & 0.005 \\
\hline ER-/PR- & & $2.67(1.92,3.73)$ & $<0.001$ & & $1.82(1.41,2.33)$ & $<0.001$ \\
\hline Therapy & $<0.001$ & & & $<0.001$ & & \\
\hline Only mastectomy & & Reference & & & Reference & \\
\hline Only partial mastectomy & & $1.80(1.22,2.66)$ & 0.003 & & $2.04(1.53,2.72)$ & $<0.001$ \\
\hline Mastectomy + radiotherapy & & $2.86(1.83,4.48)$ & $<0.001$ & & $2.19(1.50,3.20)$ & $<0.001$ \\
\hline $\begin{array}{l}\text { Partial mastectomy + } \\
\text { radiotherapy }\end{array}$ & & $0.64(0.45,0.93)$ & 0.018 & & $0.69(0.52,0.91)$ & 0.008 \\
\hline
\end{tabular}

*, American Indian/Alaska Native, Asian/Pacific Islander. BCSS, breast cancer-specific survival; OS, overall survival; CI, confidence interval; HR, hazard ratio; ER, estrogen receptor; PR, progesterone receptor. 
A

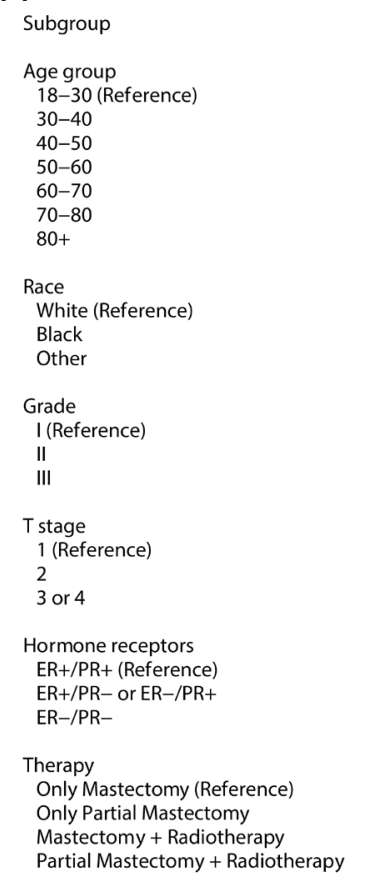

Hazard Ratio Plot of BCSS

$\begin{array}{ll}\text { No. of Patients (\%) } & \text { HR }(95 \% \mathrm{Cl}) \\ & \\ 140(1.0) & \\ 1029(7.5) & 0.77[0.17,3.44] \\ 2959(21.5) & 0.61[0.14,2.60] \\ 4235(30.8) & 0.91[0.22,3.79] \\ 3721(27.1) & 1.04[0.25,4.34] \\ 1459(10.6) & 1.63[0.39,6.90] \\ 212(1.5) & 5.22[1.19,22.83] \\ & \\ 10576(76.9) & 1.19[0.78,1.81] \\ 1506(10.9) & 0.48[0.26,0.90] \\ 1673(12.2) & \\ & \\ 639(4.6) & 3.52[0.86,14.40] \\ 5087(37.0) & 3.34[0.82,13.58] \\ 8029(58.4) & \\ & \\ 8274(60.2) & 2.05[1.49,2.82] \\ 4819(35.0) & 2.80[1.65,4.77] \\ 662(4.8) & \\ & \\ 7105(51.7) & 1.46[0.97,2.19] \\ 2779(20.2) & 2.23[1.58,3.15] \\ 3871(28.1) & \\ & \\ 5054(36.7) & 2.03[1.23[1.23,3.35] \\ 1808(13.1) & \\ 678(4.9) & \\ 6215(45.2) & \end{array}$

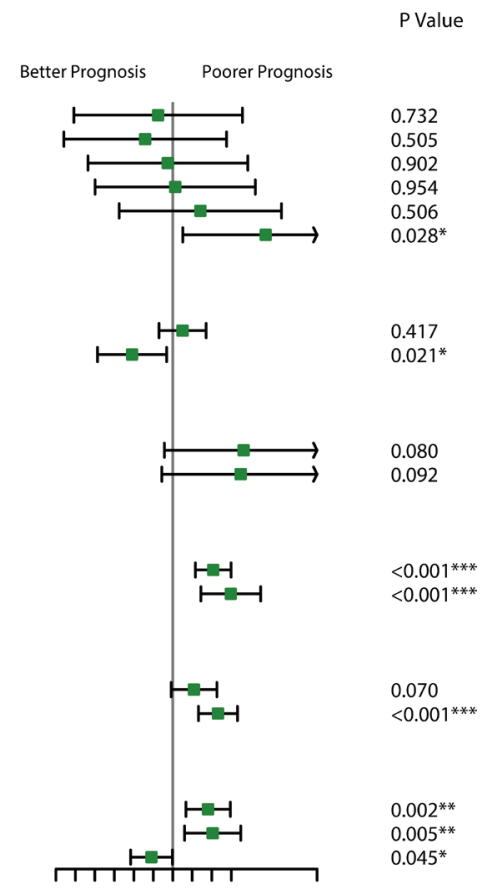

B

Subgroup
Age group
$18-30$ (Reference)
$30-40$
$40-50$
$50-60$
$60-70$
$70-80$
$80+$
Race
White (Reference)
Black
Other
Grade
I (Reference)
II
III
T stage
1 (Reference)
2
3 or 4
Hormone receptors
ER+/PR+ (Reference)
ER+/PR-or ER-/PR+
ER-/PR-
Therapy
Only Mastectomy (Reference)
Only Partial Mastectomy
Mastectomy + Radiotherapy
Partial Mastectomy + Radiotherapy

Hazard Ratio Plot of OS

\begin{tabular}{|c|c|}
\hline No. of Patients (\%) & $\mathrm{HR}(95 \% \mathrm{Cl})$ \\
\hline $\begin{array}{l}140(1.0) \\
1029(7.5) \\
2959(21.5) \\
4235(30.8) \\
3721(27.1) \\
1459(10.6) \\
212(1.5)\end{array}$ & $\begin{array}{l}0.65[0.19,2.26] \\
0.53[0.16,1.73] \\
0.85[0.27,2.72] \\
1.42[0.45,4.50] \\
2.56[0.80,8.20] \\
6.20[1.87,20.58]\end{array}$ \\
\hline $\begin{array}{l}10576(76.9) \\
1506(10.9) \\
1673(12.2)\end{array}$ & $\begin{array}{l}1.22[0.88,1.68] \\
0.60[0.39,0.93]\end{array}$ \\
\hline $\begin{array}{l}639(4.6) \\
5087(37.0) \\
8029(58.4)\end{array}$ & $\begin{array}{l}1.76[0.82,3.78] \\
1.88[0.88,4.01]\end{array}$ \\
\hline $\begin{array}{l}8274(60.2) \\
4819(35.0) \\
662(4.8)\end{array}$ & $\begin{array}{l}1.85[1.46,2.34] \\
2.38[1.55,3.66]\end{array}$ \\
\hline $\begin{array}{l}7105(51.7) \\
2779(20.2) \\
3871(28.1)\end{array}$ & $\begin{array}{l}1.28[0.96,1.71] \\
1.46[1.13,1.90]\end{array}$ \\
\hline $\begin{array}{l}5054(36.7) \\
1808(13.1) \\
678(4.9) \\
6215(45.2)\end{array}$ & $\begin{array}{l}1.95[1.45,2.61] \\
1.70[1.12,2.59] \\
0.69[0.52,0.91]\end{array}$ \\
\hline
\end{tabular}

P Value

Better Prognosis Poorer Prognosis

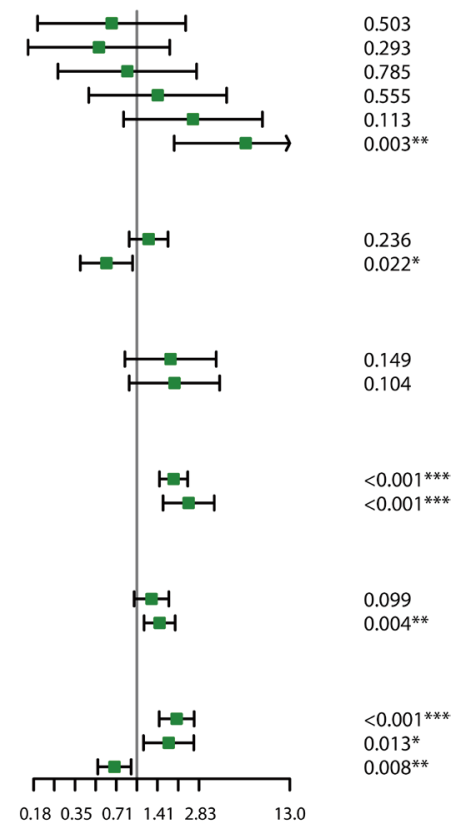

Figure 1 Forest plots of multivariate Cox analysis. (A) Breast cancer-specific survival; (B) overall survival. *, $\mathrm{P}<0.05$; ${ }^{* *}, \mathrm{P}<0.01$; ***, $\mathrm{P}<0.001$. 

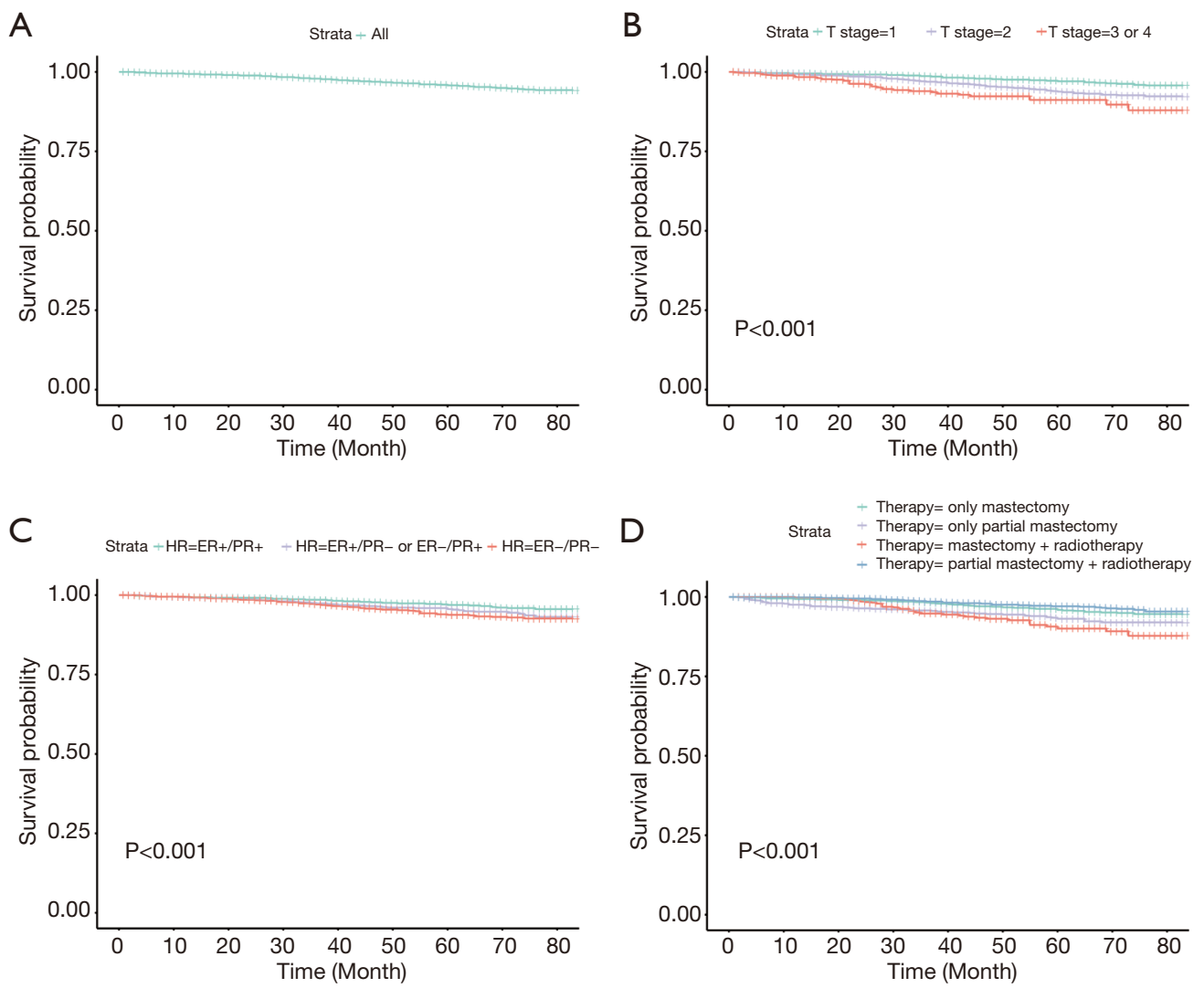

Figure 2 Kaplan-Meier overall survival curves. (A) Overall cohort; and for each prognostic factor: (B) T stage; (C) hormone receptors (HR); (D) therapy.

LN- cancer is still a blind spot for clinicians and remains to be determined $(17,19,22,29)$. Therefore, we designed this observational study to explore the high-risk factors of the $\mathrm{LN}$ - population in the single-targeted era of treatment. A total of 13,755 patients were screened from the SEER database according to our inclusion and exclusion criteria. According to the univariate and multivariate Cox analyses, some variables were significantly associated with both BCSS or OS, including age group, ethnicity, clinical T stage, HR status, and surgical type. Generally, age $\geq 80$ years was an independent risk factor associated with poor prognosis, but no differences were found among other age groups. Owing to the intrinsic high mortality rate of patients aged $\geq 80$ years, it is reasonable to believe that age is not directly related to prognosis. Differences identified between ethnicities may be related to a variety of complex social and economic factors, and were therefore not considered. $\mathrm{T}$ stage and prognosis were negatively correlated, as higher T stage was indicative of poorer BCSS and OS than lower $\mathrm{T}$ stage. Furthermore, HR status was also found to be associated with survival, wherein $\mathrm{ER}+\mathrm{PR}+$ exhibited the lowest risk among the four types, which may be due to benefits from a variety of endocrine therapies. ER+/ PR- or ER-/PR+ and ER-/PR- populations separately were associated with increased risks owing to the lack of endocrine therapy, highlighting the need for more effective anti-HER2 treatments. In terms of therapy, partial mastectomy without radiotherapy and mastectomy with radiotherapy exhibited a higher risk, while the latter may be related to higher clinical $\mathrm{T}$ stage.

To individually predict the survival of the HER2+ and $\mathrm{LN}$ - populations, a Cox proportional hazards regression model was applied to construct the nomogram, and the performance of the nomogram was evaluated by internal bootstrap validation and displayed using calibration curves. In our model, the calibration curves demonstrated good consistency between the estimated and actual probabilities for both BCSS and OS. On the other hand, the C-index values were $>0.75$ in both BCSS and OS, which also confirmed the high credibility of the model. 


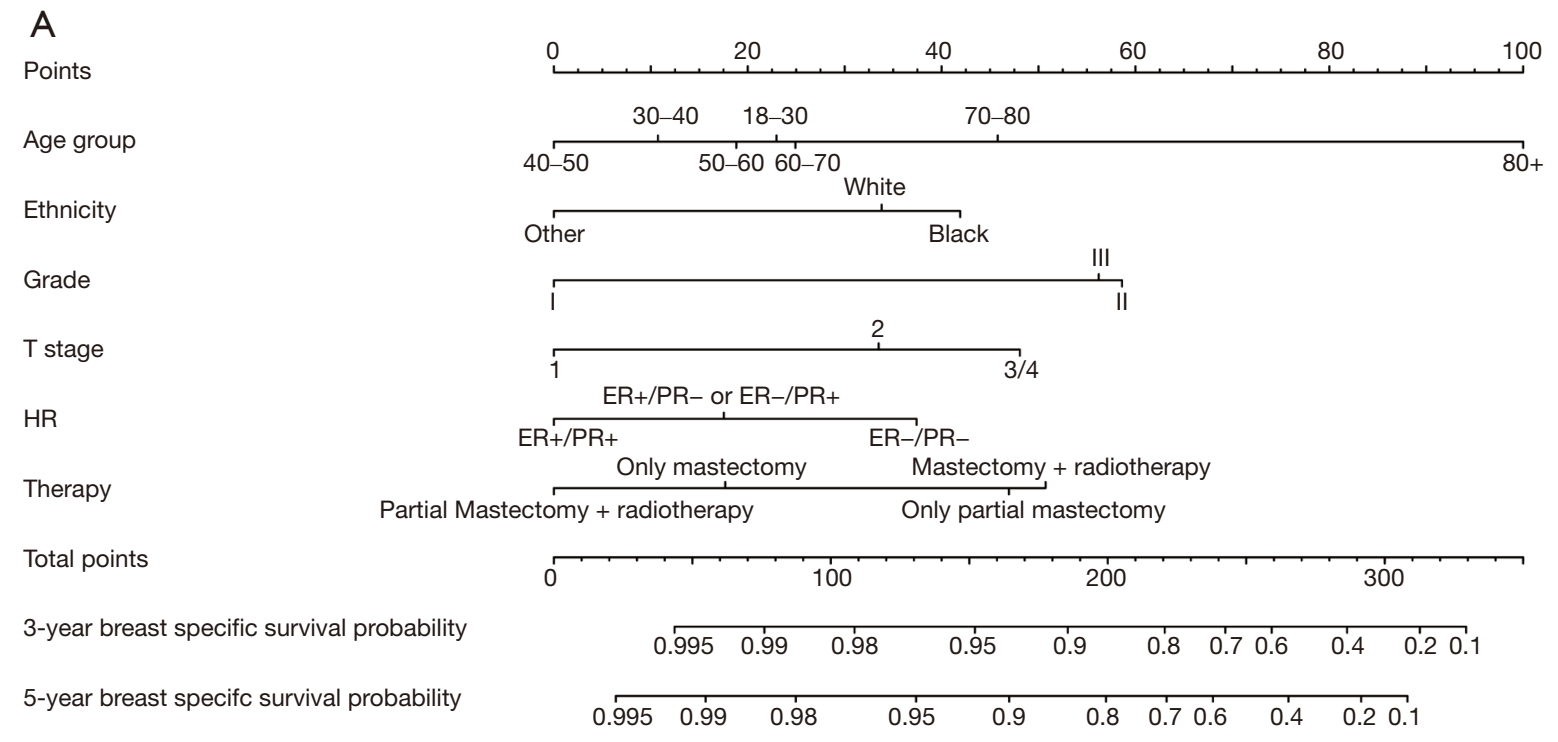

\section{B}

Points

0

20

40

60

80

100

Age group

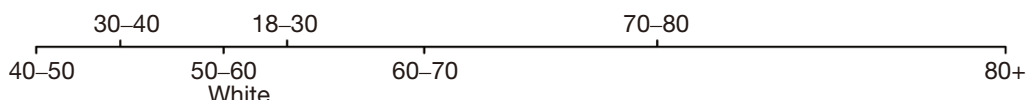

Ethnicity

Grade

T stage

HR

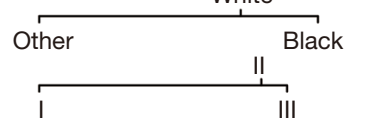

e

Therapy

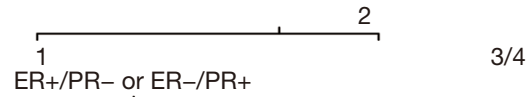

ER+/PR- or ER-/PR+

$\mathrm{ER}+\overparen{P R+} \begin{gathered}\mathrm{ER}-/ \mathrm{PR}- \\ \text { Only mastectomy }\end{gathered}$

Total points

3-year breast specific survival probability

5-year breast specifc survival probability

Partial mastectomy + radiotherapy Mastectomy + radiotherapy

\begin{tabular}{|c|c|c|c|c|c|c|c|c|c|c|}
\hline 0 & 40 & & 80 & & 120 & 160 & & 200 & & 240 \\
\hline 0.995 & 0.99 & 0.98 & 0.97 & 0.95 & 0.9 & 0.8 & 0.7 & 0.6 & 0.4 & \\
\hline 0.9 & 0.98 & 0.97 & 0.95 & & 0.9 & 0.7 & 0.6 & 0.4 & 0.2 & 0.1 \\
\hline
\end{tabular}

Figure 3 Nomograms for predicting the 3-and 5-year survival. (A) Breast cancer-specific survival; (B) overall survival. Each variable corresponds to a score on the points scale. After adding up the total points, the predicted survival possibility could be obtained by projecting the total points to the survival axis.

This nomogram can be used in clinical practice to evaluate the individual survival risk of HER2 $+\mathrm{LN}$ - breast cancer according to clinicopathological variables, and patients with high total scores in the nomogram (T3 or T4 stage, ER-/PR-, partial mastectomy without radiotherapy) can be recommended for dual-targeted therapy, including trastuzumab combined with pertuzumab or TKIs, or sequential TKIs after trastuzumab therapy.

Despite its novelty, our study still has some limitations that should be noted. Firstly, given the nature of retrospective research, a high potential for selection bias and a lack of standardized specimen handling were inevitable. 
A

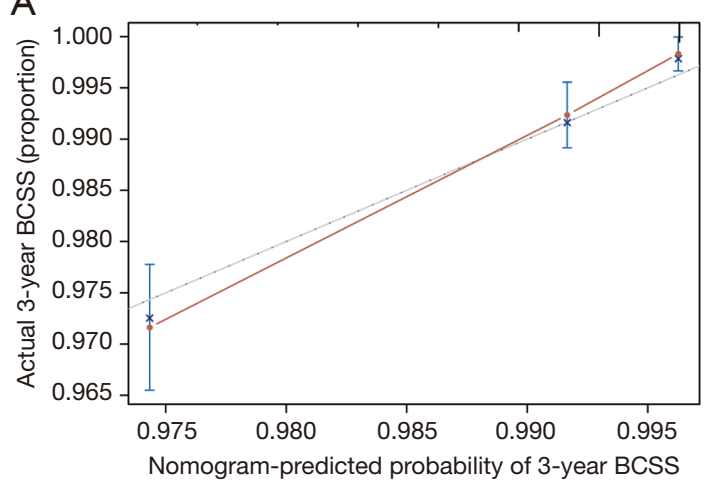

C

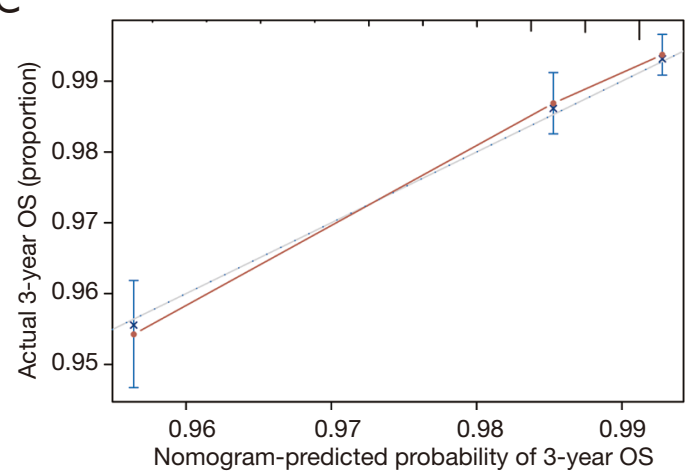

B
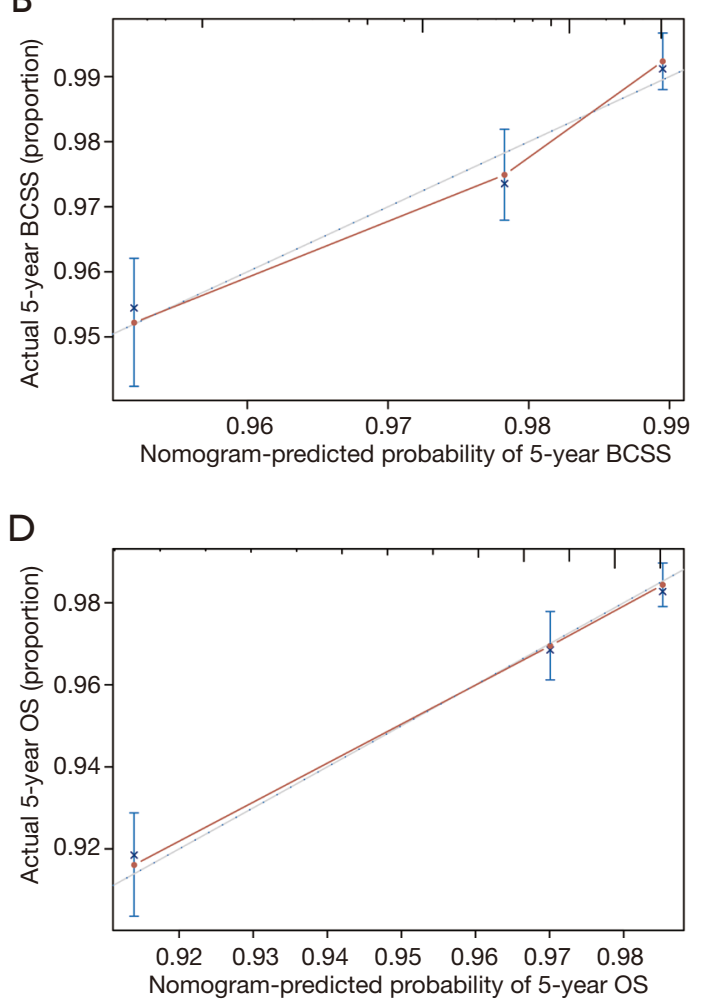

Figure 4 Calibration curves for the nomogram-predicted probability of 3- and 5-year survival. (A,B) BCSS; (C,D) OS. Nomogrampredicted survival probability is plotted on the $\mathrm{X}$-axis and actual probability is plotted on the $\mathrm{Y}$-axis. The dotted red line (plotted by function $\mathrm{y}=\mathrm{x})$ indicates the perfect match of actual and predicted results. BCSS, breast cancer-specific survival; OS, overall survival.

Secondly, only internal bootstrap validation was applied due to the lack of databases with sufficient information. The model would be more robust and persuasive with external validation. Further prospective studies are encouraged to improve and verify our models.

\section{Conclusions}

Higher clinical T stage, HR- status, and partial mastectomy without radiotherapy are independent risk factors for BCSS and $\mathrm{OS}$ in patients with HER2+ and $\mathrm{LN}$ - breast cancer. In this study, prognostic nomograms targeting this population were established and validated, which could be useful in clinical counseling. In clinical practice, patients can be recommended for single-targeted $(\mathrm{H})$ or dual-targeted (trastuzumab plus pertuzumab or TKIs, or sequential TKIs after trastuzumab) therapy according to individualized factors.

\section{Acknowledgments}

Funding: This study was supported by grants from the Program of National Key Clinical Specialist Construction (No. 413F1Z113), Military Medical Staff Innovation Plan of Southwest Hospital (No. SWH2018BJLC-04) and Army Medical University (No. XZ-2019-505-042).

\section{Footnote}

Reporting Checklist: The authors have completed the TRIPOD reporting checklist. Available at https://dx.doi. org/10.21037/gs-21-392

Conflicts of Interest: All authors have completed the ICMJE uniform disclosure form (available at https://dx.doi. org/10.21037/gs-21-392). The authors have no conflicts of interest to declare. 
Ethical Statement: The authors are accountable for all aspects of the work in ensuring that questions related to the accuracy or integrity of any part of the work are appropriately investigated and resolved. The study was conducted in accordance with the Declaration of Helsinki (as revised in 2013).

Open Access Statement: This is an Open Access article distributed in accordance with the Creative Commons Attribution-NonCommercial-NoDerivs 4.0 International License (CC BY-NC-ND 4.0), which permits the noncommercial replication and distribution of the article with the strict proviso that no changes or edits are made and the original work is properly cited (including links to both the formal publication through the relevant DOI and the license). See: https://creativecommons.org/licenses/by-nc-nd/4.0/.

\section{References}

1. Slamon DJ, Clark GM, Wong SG, et al. Human breast cancer: correlation of relapse and survival with amplification of the HER-2/neu oncogene. Science 1987;235:177-82.

2. Takada M, Toi M. Neoadjuvant treatment for HER2positive breast cancer. Chin Clin Oncol 2020;9:32.

3. Cameron D, Piccart-Gebhart MJ, Gelber RD, et al. 11 years' follow-up of trastuzumab after adjuvant chemotherapy in HER2-positive early breast cancer: final analysis of the HERceptin Adjuvant (HERA) trial. Lancet 2017;389:1195-205.

4. Marty M, Cognetti F, Maraninchi D, et al. Randomized phase II trial of the efficacy and safety of trastuzumab combined with docetaxel in patients with human epidermal growth factor receptor 2-positive metastatic breast cancer administered as first-line treatment: the M77001 study group. J Clin Oncol 2005;23:4265-74.

5. Lee HY, Shin IS, Rim CH. Benefits of adjuvant treatment including trastuzumab in HER2-positive pT1a-bN0M0 breast cancer: a systematic review and meta-analysis. Ann Transl Med 2020;8:187.

6. Gianni L, Eiermann W, Semiglazov V, et al. Neoadjuvant chemotherapy with trastuzumab followed by adjuvant trastuzumab versus neoadjuvant chemotherapy alone, in patients with HER2-positive locally advanced breast cancer (the NOAH trial): a randomised controlled superiority trial with a parallel HER2-negative cohort. Lancet 2010;375:377-84.

7. Gong DH, Ge JY, Chen YY, et al. HER2 overexpression in ductal carcinoma in situ is associated with ipsilateral breast cancer recurrence after conservative surgery. Transl Cancer Res 2020;9:3787-93.

8. Cortés J, Ciruelos E, Pérez-García J, et al. Contextualizing pertuzumab approval in the treatment of HER2-positive breast cancer patients. Cancer Treat Rev 2020;83:101944.

9. Slamon DJ, Leyland-Jones B, Shak S, et al. Use of chemotherapy plus a monoclonal antibody against HER2 for metastatic breast cancer that overexpresses HER2. N Engl J Med 2001;344:783-92.

10. Piccart-Gebhart MJ, Procter M, Leyland-Jones B, et al. Trastuzumab after adjuvant chemotherapy in HER2positive breast cancer. N Engl J Med 2005;353:1659-72.

11. Romond EH, Perez EA, Bryant J, et al. Trastuzumab plus adjuvant chemotherapy for operable HER2-positive breast cancer. N Engl J Med 2005;353:1673-84.

12. Vogel CL, Cobleigh MA, Tripathy D, et al. Efficacy and safety of trastuzumab as a single agent in first-line treatment of HER2-overexpressing metastatic breast cancer. J Clin Oncol 2002;20:719-26.

13. Leyland-Jones B. Trastuzumab: hopes and realities. Lancet Oncol 2002;3:137-44.

14. Slamon D, Eiermann W, Robert N, et al. Adjuvant trastuzumab in HER2-positive breast cancer. N Engl J Med 2011;365:1273-83.

15. Slamon DJ, Eiermann W, Robert NJ, et al. Abstract S5-04: Ten year follow-up of BCIRG-006 comparing doxorubicin plus cyclophosphamide followed by docetaxel (AC T) with doxorubicin plus cyclophosphamide followed by docetaxel and trastuzumab (AC TH) with docetaxel, carboplatin and trastuzumab (TCH) in HER2 + early breast cancer. Cancer Res 2016;76:Abstract S5-04.

16. Wang J, Xu B. Targeted therapeutic options and future perspectives for HER2-positive breast cancer. Signal Transduct Target Ther 2019;4:34.

17. von Minckwitz G, Procter M, de Azambuja E, et al. Adjuvant Pertuzumab and Trastuzumab in Early HER2Positive Breast Cancer. N Engl J Med 2017;377:122-31.

18. Howie LJ, Scher NS, Amiri-Kordestani L, et al. FDA Approval Summary: Pertuzumab for Adjuvant Treatment of HER2-Positive Early Breast Cancer. Clin Cancer Res 2019;25:2949-55.

19. Martine P, Marion P, Debora F, et al. editors. GS1-04. Interim overall survival analysis of APHINITY (BIG 4-11): A randomized multicenter, double-blind, placebocontrolled trial comparing chemotherapy plus trastuzumab plus pertuzumab versus chemotherapy plus trastuzumab plus placebo as adjuvant therapy in patients with operable 
HER2-positive early breast cancer. San Antonio Breast Cancer Symposium, 2019.

20. Chan A, Delaloge S, Holmes FA, et al. Neratinib after trastuzumab-based adjuvant therapy in patients with HER2-positive breast cancer (ExteNET): a multicentre, randomised, double-blind, placebo-controlled, phase 3 trial. Lancet Oncol 2016;17:367-77.

21. Martin M, Holmes FA, Ejlertsen B, et al. Neratinib after trastuzumab-based adjuvant therapy in HER2-positive breast cancer (ExteNET): 5-year analysis of a randomised, double-blind, placebo-controlled, phase 3 trial. Lancet Oncol 2017;18:1688-700.

22. Chan A, Moy B, Mansi J, et al. Final Efficacy Results of Neratinib in HER2-positive Hormone Receptor-positive Early-stage Breast Cancer From the Phase III ExteNET Trial. Clin Breast Cancer 2021;21:80-91.e7.

23. Surveillance, Epidemiology, and End Results (SEER) Program. Available online: https://seer.cancer.gov/

24. Pusztai L, Foldi J, Dhawan A, et al. Changing frameworks in treatment sequencing of triple-negative and HER2positive, early-stage breast cancers. Lancet Oncol

Cite this article as: Shi Q, Wang J, Ai X, Xuhong J, Ma D, Zhang Y, Qi X, Jiang J. Development and validation of a prognostic nomogram for early HER2-positive and lymph node-negative breast cancer. Gland Surg 2021;10(7):2255-2265. doi: $10.21037 / g s-21-392$ 2019;20:e390-e396.

25. Waks AG, Winer EP. Breast Cancer Treatment: A Review. JAMA 2019;321:288-300.

26. Lubsen J, Pool J, van der Does E. A practical device for the application of a diagnostic or prognostic function. Methods Inf Med 1978;17:127-9.

27. Harrell FE, Lee KL, Mark DB. Multivariable prognostic models: issues in developing models, evaluating assumptions and adequacy, and measuring and reducing errors. Stat Med 1996 Feb;15:361-87.

28. DeSantis CE, Ma J, Gaudet MM, et al. Breast cancer statistics, 2019. CA Cancer J Clin 2019;69:438-51.

29. Nadia H, Seock-Ah I, Carlos HB, et al. editors. Primary analysis of KAITLIN: A phase III study of trastuzumab emtansine (T-DM1) + pertuzumab versus trastuzumab + pertuzumab + taxane, after anthracyclines as adjuvant therapy for high-risk HER2-positive early breast cancer (EBC)2020; ASCO Virtual Scientific Program: American Society of Clinical Oncology.

(English Language Editor: A. Kassem) 


\section{Supplementary}

Table S1 Clinicopathologic characteristics' distribution of different therapy groups

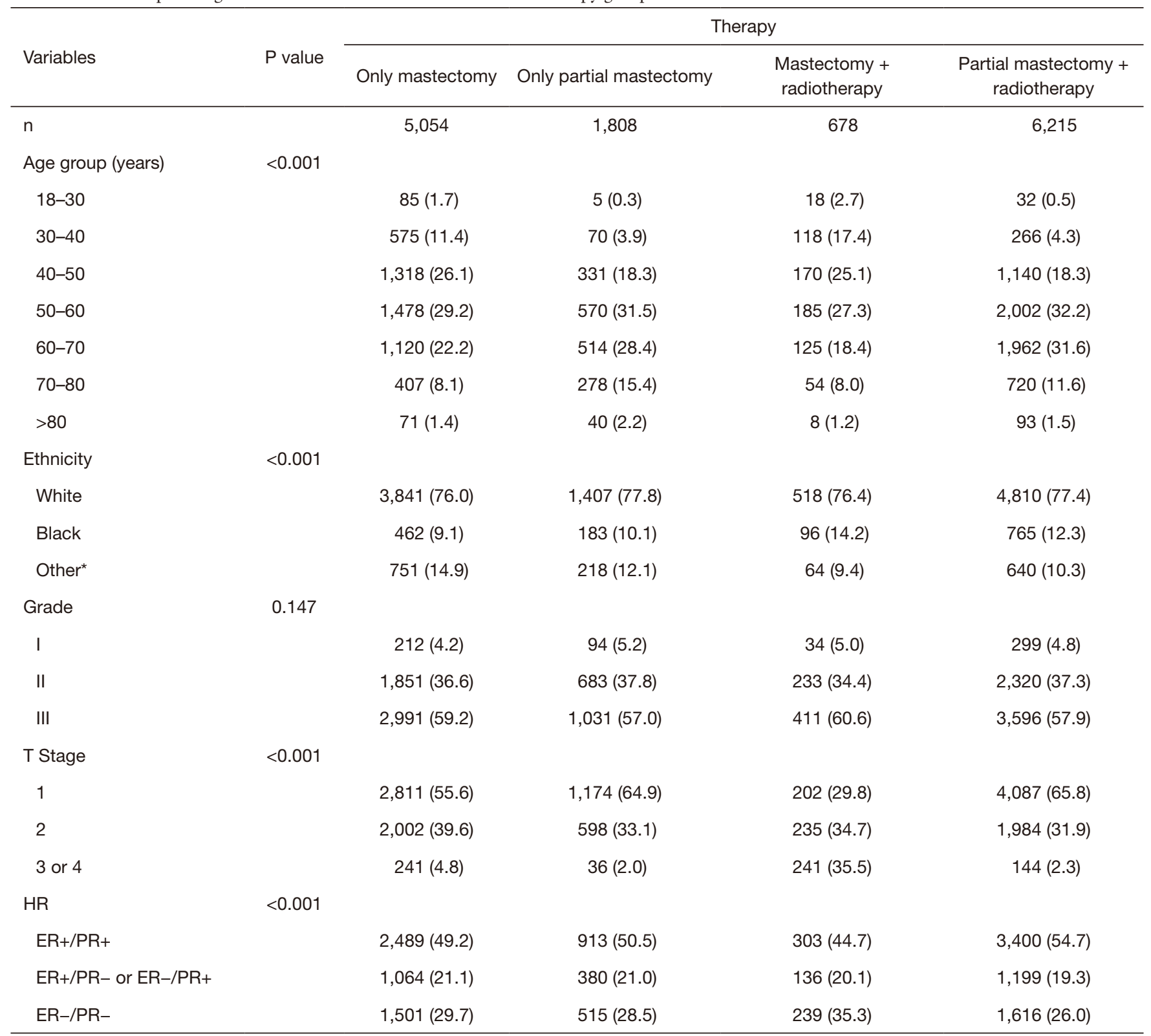

*, American Indian/Alaska Native, Asian/Pacific Islander. HR, hazard ratio; ER, estrogen receptor; PR, progesterone receptor. 